\title{
International Conference on Modelling, Computation and Optimization in Information Systems and Management Sciences
}

\author{
Hoai An Le Thi · Joaquim Judice
}

Published online: 27 September 2011

(C) Springer Science+Business Media, LLC 2011

This special issue is dedicated to the International Conference on "Modelling, Computation and Optimization in Information Systems and Management Sciences" (MCO) which took place at University Paul Verlaine-Metz, France and University of Luxembourg on September 8-10, 2008. MCO 2008 was the second event in the series of MCO conferences organized by LITA, the Laboratory of Theoretical and Applied Computer Science, University Paul Verlaine-Metz, France every four years. The conference was attended by approximately 120 scientists from 27 countries. The scientific program consisted of 6 invited plenary speakers and of the oral presentation of 65 selected full papers, as well as 34 selected abstracts covering all main topic areas. The refereed Proceedings of MCO 2008 including 65 selected papers were published in a book series Communications in Computer and Information Science (CCIS) of Springer.

This MCO 2008 special issue includes high quality contributions on recent developments in optimization techniques and its applications for solving real world problems arising from economics and management sciences. In the first paper "On solving Linear Complementarity Problems by DC programming and DCA", the authors investigate a new and efficient local optimization approach based on DC (Difference of Convex functions) programming and DCA (DC Algorithms) for solving one of the fundamental problems of mathematical programming which has many applications in several areas of science, engineering and economics. Also based on DC programming and DCA, the authors of the second paper "An Efficient DC Programming Approach for Portfolio Decision with Higher Moments" propose an efficient

H.A. Le Thi $(\bowtie)$

Paul Verlaine University, Metz, France

e-mail: lethi@univ-metz.fr

J. Judice

University of Coimbra, Coimbra, Portugal 
method for an application in portfolio selection which is modelled as a NP-hard nonconvex polynomial optimization problem. In the third paper "Genetic Tabu Search for robust channel assignment under dynamic traffic data", the authors introduce a new channel assignment model for GSM radio networks and develop an original and effective hybrid algorithm to get high quality frequency plans. A problem of lot-sizing and sequencing several products on a single machine is studied in the fourth paper "Multi-Product Lot-Sizing and Sequencing on a Single Imperfect Machine", where efficient based FPTAS algorithms are proposed.

We feel that this special issue will be a scientific source of information to researchers in optimization, computational engineering, computer science and applied mathematics.

We would like to thank all the persons that have contributed to the success of the conference and to this special issue, in particular to the authors of the papers and to the other members of the scientific committee and the referees, for their helpful comments on these papers. Finally, the interest of the sponsors in the meeting and their assistance are gratefully acknowledged.

We thank Professor William Hager, the Editor of Journal of Computational Optimization and Applications for hosting the special issue volume, and Springer for publishing the volume.

The Guest Editors 